\title{
A Prognostic Nomogram via Competing Risk Model with Patterns of Extracranial Metastasis in Elderly NSCLC Patients with Synchronous Brain- metastasis: A Large Population-based Study
}

\section{Mingwei Zhang}

The First Affiliated Hospital of Fujian Medical University

Zijing Zhu

Fujian Normal University

Wenying Jiang

The Third Affiliated Hospital of Soochow University

\section{Shaoli Peng}

The First Affiliated Hospital of Fujian Medical University

\section{Weitong Zhou}

The First Affiliated Hospital of Fujian Medical University

Jinfu Zhuang

The First Affiliated Hospital of Fujian Medical University

Jinsheng Hong

The First Affiliated Hospital of Fujian Medical University

Xiaoping Chen ( $\nabla$ xpchen@fjnu.edu.cn )

Fujian Normal University

\section{Research Article}

Keywords: NSCLC, brain metastasis, extracranial metastasis, geriatric population, competing risk model

Posted Date: February 18th, 2022

DOI: https://doi.org/10.21203/rs.3.rs-1357913/v1

License: (c) (1) This work is licensed under a Creative Commons Attribution 4.0 International License. Read Full License 


\section{Abstract}

Introduction: To determine whether the patterns of extracranial metastasis (ECMs) provide supplementary prognositc information to DS-GPA in elderly NSCLC patients with synchronous BM.

Methods: This study included 4974 NSCLC patients with initial BM diagnosed from 2010 to 2015 using the Surveillance Epidemiology and End Results (SEER) program. Patients were divided randomly into training and hold-out test sets. Patterns of ECMs were established based on the difference of survival via competing risk analysis in the training set. A nomogram prediction of 6-month, 12-month, and 18-month disease-specific survival (DSS) was built using independent prognostic factors.

Results: Three patterns of ECM were recognized: MA (neither liver, bone, nor lung involvement), MB (without liver involvement), and MC (with liver involvement). Comparing MA, MB and MC showed significant correlation to survival (SHR, 1.126, 95\% Cl, 1.053-1.205, P<0.001; SHR, 1.46, 95\% Cl, 1.339$1.592, \mathrm{P}<0.001$, respectively). In the hold-out test set, the AUC of the ROC curve for the 6 -month DSS prediction reached 0.778 , whereas reaching 0.757 in the training set. The calibration curves did not deviate from the reference line. Decision curve analyses revealed the net benefit of the nomogram for clinical utility.

Conclusions: These results help clinicians make decisions for brain-metastatic NSCLC in the era of precision therapy. The risk stratification of extracranial involvements indicates differential treatment for elderly NSCLC patients with synchronous brain-metastasis.

\section{Introduction}

Globally, lung cancer (LC) is the number one cause of cancer-associated death[1]. Most LC-related deaths are due to metastasis, with brain being a common site. Non-small cell lung cancer (NSCLC) is the most prevalent subtype and comprises about $85 \%$ of cases[2]. The incidence of brain metastasis (BM) in NSCLC is growing due to imaging technique advancements, improved survival from novel regimens and population aging[3]. The conventional therapies for NSCLC with BM include surgery, radiation and chemotherapy with a median overall survival (mOS) ranging from 4.0 to 31.0 months[4]. The introduction of tyrosine kinase inhibitors and the immune checkpoint inhibitors has improved the 5-year survival rate from $30-60 \%$ in selected patients with metastatic NSCLC[5]. NSCLC with de novo BM has been recognized as an entity with heterogeneous prognosis[6] and its risk stratification is a critical component of standard management.

Several prognostic models have been applied to predict BM patients' outcomes, such as the DiseaseSpecific Graded Prognostic Assessment (DS-GPA). Besides age, DS-GPA considers four clinical factors: Karnofsky Performance Status (KPS) [7], extracranial metastasis (ECM) and BM lesions. Genomic and proteomic biomarkers have been reported to stratify patients, which necessitates invasive biopsy and costly molecular sequencing[6]. The invasive nature of biopsy and the high cost of genomic tests limit 
their large-scale application. Meanwhile, utilizing readily available clinical data, recombination of the M1 stage for stage IV breast cancer can forecast prognosis and effects of primary surgery better[8].

This study on breast cancer has added additional details to DS-GPA, but no such research to modify DSGPA has been conducted for NSCLC yet. While more ECM lesions have proven to have a worse prognosis in NSCLC patients with BM [9], the subdivision of ECM, other than the total number, might more sufficiently address the medical challenges in NSCLC with BM. Noteworthy, half NSCLC patients are over 70-years-old[10]. Current epidemiological trends make LC in the geriatric population increasingly recognized as a public health concern[11]. Yet, their prognostic estimates mainly stem from younger, healthier patients enrolled in clinical trials[12], because patients over 70 years old have historically been excluded from major landmark trials[13]. According to the WHO and published estimation, cancer is not the number one cause of death in people older than 70-years[14]. Compared with the Cox proportional hazards model, the competing risk model improves the stratification of senior patients with cancer by the risk of dying from cancer relative to overall mortality[15].

A hospital-based cohort study of elderly NSCLC patients with de novo BM would be extremely challenging, regarding to obtain samples sufficient to detect ECM patterns' impact on survival. Due to limitations of registries, the incidence of BM remains indistinct. Yet, the Surveillance Epidemiology and End Results (SEER) program revealed data relevant to with or without BM at diagnosis of malignancies in 2016[16]. Given that it is a large population-based database, selection bias is largely reduced. Exploiting NSCLC cohorts in SEER datasets, we analyzed the prognostic value of the ECM patterns in septuagenarians and octogenarians with de novo BM and plotted a nomogram for clinical usage.

\section{Materials And Methods \\ 2.1 Patients}

The study data set was retrieved from the SEER cancer registries, which are freely accessible via SEER ${ }^{\star}$ Stat 8.3.8 (https://seer.cancer.gov). The SEER registry comprises 18 cancer registries across America, taking up about $30 \%$ of the US population. The SEER data are periodically updated with clinical information, such as tumor basics, therapies, secondary tumors, demographics and cause of death. The present study was conducted in compliance with the Helsinki Declaration. Given that the SEER registry contains only de-identified information, ethical review and informed consent requirements were waived.

Following the ICD-0-3, all lung cancer cases (site codes C34.0-34.8) diagnosed between 2010 and 2015 were potentially included in our study. Restrictive inclusion items were applied and were as follows: 1 ) NSCLC (histology codes, 8012/ 8013/ 8014/ 8046/ 8052/ 8070-8078/ 8083/ 8084/ 8140/ 8141/ 8143/ 8144/ 8146/ 8147/ 8250-8255/ 8260/ 8310/ 8323/ 8481/ 8560); 2) malignant behavior; 3) microscopically confirmed primary lung cancer; 4) diagnosed between 2010 and 2015, 5) de novo BM cases; 3) AJCC T1 to Tx stages. 
Given that elderly metastatic lung cancer patients rarely underwent surgeries, those who received surgery $(<5 \%)$ were excluded from this study to improve the homogeneity of the study population. Other exclusion criteria were: 1 ) aged younger than 70 years; 2 ) with prior or subsequent cancers; 3 ) underwent surgical treatments; 4) less than one month of follow-up; 5) patients with unavailable information regarding survival duration, status, cause of death or other important items.

We included clinical characteristics, such as gender, race, age, American Joint Committee on Cancer (AJCC) T and N stage, histologic type, chemoradiotherapy, and ECM involvement. The included patients were then randomly divided into a training and a hold-out test set in a 50/50 ratio. The former was used to train the weights of the model, while the latter was to validate the predictive ability of the trained model.

\subsection{Statistics}

Based on the accessibility of the SEER databank, ECM involvement in de novo brain-metastatic NSCLC patients were separated into eight categories: none, bone alone, liver alone, lung alone, both bone and liver, bone and lung, liver and lung, or all of the three. The main outcome was disease-specific survival (DSS), and a competing risk is an event whose occurrence precludes the occurrence of the primary event of interest.

The univariable and multivariable analyses of the competing risk model were conducted via Fine and Gray's regression, which led to the recombination of extracranial involvements for further investigation. Multivariable analysis of the competing risk model was recalculated in the training, test, and whole sets, followed by subgroup analysis and interaction tests. Multivariate analysis of overall survival (OS) was carried out via Cox proportional hazards model. Sensitivity analyses were done to support the primary analysis. Cumulative incidence curves for DSS and OS were estimated via Gray's and log-rank tests, respectively.

A nomogram of independent factors predicting DSS was depicted based on multivariate analysis of the competing risk model. A stepwise variable selection method was applied. For calibration, estimated probabilities were contrasted with the actual ones. Time-dependent ROC curves were used to judge discrimination. Decision curves analysis (DCA) was applied to visualize the clinical benefits of alternative models by calculating net benefits under different threshold probabilities. Curves of therapy plan (highest cost) and no plan (no benefit) were used as references.

Chi-square and Fisher exact test were used for categorical data. Statistical significance level was set at a $P$ value (two-tailed) $<0.05$, except for those that were specifically stated. Chi-square, Fisher exact test, Cox proportional hazards model, Kaplan-Meier method, Gray's test, and Fine-Gray regression model were performed using R (version 3.4.3; The R foundation for statistics, Vienna, Austria).

\section{Results}




\section{1 characteristics}

In total, 4,974 cases were identified as de novo brain-metastatic NSCLC aged over 70 years, with both training and hold-out test sets allocated 2,487 cases each. The most frequently involved organs were bone, contra-lateral lung and liver. Clinicopathologic characteristics were well-balanced between the two data sets. Specific basic characteristics are outlined in Table 1 and supplemental Table 1. 
Table 1

Baseline Demographic Data

\begin{tabular}{|c|c|c|c|c|}
\hline & Total $(n=4974)$ & Train $(n=2487)$ & Test $(n=2487)$ & $P$ value \\
\hline Age, n (\%) & & & & 0.588 \\
\hline$<=74$ & $2246(45)$ & $1113(45)$ & $1133(46)$ & \\
\hline$>74$ & 2728 (55) & $1374(55)$ & 1354 (54) & \\
\hline Race, n (\%) & & & & 0.196 \\
\hline Black & 460 ( 9$)$ & 230 (9) & $230(9)$ & \\
\hline White & 3954 (79) & 1997 (80) & 1957 (79) & \\
\hline Others & $560(11)$ & $260(10)$ & 300 (12) & \\
\hline Sex, n (\%) & & & & 0.461 \\
\hline Male & 2451 (49) & $1212(49)$ & 1239 (50) & \\
\hline Female & $2523(51)$ & $1275(51)$ & $1248(50)$ & \\
\hline AJCC.T, n (\%) & & & & 0.203 \\
\hline T1 & $532(11)$ & $256(10)$ & $276(11)$ & \\
\hline T2 & $1362(27)$ & $697(28)$ & $665(27)$ & \\
\hline T3 & 1103 (22) & $558(22)$ & $545(22)$ & \\
\hline $\mathrm{T} 4$ & $1400(28)$ & $671(27)$ & 729 (29) & \\
\hline Tx & $577(12)$ & $305(12)$ & $272(11)$ & \\
\hline AJCC.N, n (\%) & & & & 0.146 \\
\hline NO & $1188(24)$ & $565(23)$ & $623(25)$ & \\
\hline N1 & 419 ( 8) & 199 ( 8) & 220 ( 9) & \\
\hline N2 & $2225(45)$ & $1131(45)$ & $1094(44)$ & \\
\hline N3 & 844 (17) & $431(17)$ & 413 (17) & \\
\hline$N x$ & 298 ( 6) & $161(6)$ & 137 ( 6) & \\
\hline Histology, n (\%) & & & & 0.669 \\
\hline$A C$ & 3574 (72) & $1775(71)$ & 1799 (72) & \\
\hline SSC & $756(15)$ & $380(15)$ & $376(15)$ & \\
\hline
\end{tabular}

Abbreviations: AC, adenocarcinoma; SSC, squamous carcinoma; NSCLC, non-small cell lung carcinoma, nothing otherwise special. 


\begin{tabular}{|c|c|c|c|c|}
\hline & Total $(n=4974)$ & Train $(n=2487)$ & Test $(n=2487)$ & $P$ value \\
\hline NSCLC & $644(13)$ & $332(13)$ & $312(13)$ & \\
\hline \multicolumn{4}{|c|}{ Chemo-radiotherapy, n (\%) } & 0.633 \\
\hline None & $880(18)$ & $425(17)$ & $455(18)$ & \\
\hline Radiation & $1762(35)$ & $890(36)$ & $872(35)$ & \\
\hline Chemotherapy & 384 ( 8) & 199 ( 8) & 185 ( 7$)$ & \\
\hline Both & $1948(39)$ & 973 (39) & 975 (39) & \\
\hline \multicolumn{4}{|c|}{ Chemo-radiotherapy_2, n (\%) } & 0.281 \\
\hline None & $880(18)$ & $425(17)$ & $455(18)$ & \\
\hline Yes & $4094(82)$ & $2062(83)$ & $2032(82)$ & \\
\hline \multicolumn{4}{|c|}{ Bone involvement, n (\%) } & 0.386 \\
\hline None & $3274(66)$ & $1652(66)$ & $1622(65)$ & \\
\hline Yes & $1700(34)$ & $835(34)$ & $865(35)$ & \\
\hline \multicolumn{4}{|c|}{ Liver involvement, n (\%) } & 0.532 \\
\hline None & $4070(82)$ & $2026(81)$ & $2044(82)$ & \\
\hline Yes & $904(18)$ & $461(19)$ & $443(18)$ & \\
\hline \multicolumn{4}{|c|}{ Lung involvement, n (\%) } & 0.448 \\
\hline None & $3589(72)$ & $1782(72)$ & $1807(73)$ & \\
\hline Yes & $1385(28)$ & $705(28)$ & $680(27)$ & \\
\hline \multicolumn{5}{|c|}{$\begin{array}{l}\text { Abbreviations: AC, adenocarcinoma; SSC, squamous carcinoma; NSCLC, non-small cell lung } \\
\text { carcinoma, nothing otherwise special. }\end{array}$} \\
\hline
\end{tabular}

As depicted in Table 2, univariate analysis indicated that involvement of the liver, whether alone, with concomitant bone or lung metastasis, or with concomitant both bone and lung involvement was correlated with worse DSS (SHR, 1.43, 95\% Cl, 1.17-1.76, P<0.01; SHR, 1.30, 95\% Cl, 1.09-1.55, P< 0.01 ; SHR, 1.46, 95\% Cl, 1.16-1.85 P<0.01; SHR, 1.33, 95\% Cl, 1.08-1.64, $\mathrm{P}<0.01$, respectively). Multivariable analysis confirmed that these four patterns of distant metastasis were independent prognostic factors (SHR, 1.42, 95\% Cl, 1.15-1.76, P< 0.01; SHR, 1.35, 95\% Cl, 1.10-1.65, P< 0.01; SHR, 1.47, 95\% Cl, 1.17$1.85, \mathrm{P}<0.01$; SHR, $1.40,95 \% \mathrm{Cl}, 1.13-1.74, \mathrm{P}<0.01$, respectively). Regarding bone involvement alone, it was associated with worse DSS. Although its univariate analysis is not statistically significant (SHR, 1.09, 
$95 \% \mathrm{Cl}, 1.04-1.22, \mathrm{P}=0.16)$, multivariable analysis gained significance $(\mathrm{SHR}, 1.18,95 \% \mathrm{Cl}, 1.05-1.33, \mathrm{P}$ 0.01). However, no significant difference was observed with contra-lateral lung metastasis alone (SHR, $1.11,95 \% \mathrm{Cl}, 0.98-1.24, \mathrm{P}=0.08 ; \mathrm{SHR}, 1.07,95 \% \mathrm{Cl}, 0.93-1.21, \mathrm{P}=0.35$, respectively). 
Table 2

Univariate and Multivariate Analysis via Competing Risk Regression Model

Univariate Analysis

$\operatorname{SHR}(95 \% \mathrm{Cl})$

$P$ value

Multivariate Analysis

\begin{tabular}{|c|c|c|c|c|}
\hline & $\operatorname{SHR}(95 \% \mathrm{Cl})$ & $P$ value & $\operatorname{SHR}(95 \% \mathrm{Cl})$ & $P$ value \\
\hline \multicolumn{5}{|l|}{ Age.cat } \\
\hline$<=74$ & Ref & & Ref & \\
\hline$>74$ & $1.1(1.02-1.19)$ & 0.01 & $1.06(0.97-1.15)$ & 0.19 \\
\hline \multicolumn{5}{|l|}{ Race } \\
\hline Black & Ref & & Ref & \\
\hline White & $0.977(0.86-1.11)$ & 0.73 & $1.01(0.88-1.17)$ & 0.87 \\
\hline Others & $0.801(0.67-0.95)$ & 0.01 & $0.85(0.70-1.03)$ & 0.09 \\
\hline \multicolumn{5}{|l|}{ Sex } \\
\hline Male & Ref & & Ref & \\
\hline Female & $0.917(0.85-0.99)$ & 0.03 & $0.96(0.88-1.04)$ & 0.31 \\
\hline \multicolumn{5}{|l|}{ AJCC.T } \\
\hline T1 & Ref & & Ref & \\
\hline T2 & $1.17(1.01-1.35)$ & 0.03 & $1.17(1.01-1.35)$ & 0.04 \\
\hline T3 & $1.29(1.11-1.49)$ & $<0.01$ & $1.24(1.06-1.46)$ & $<0.01$ \\
\hline T4 & $1.27(1.10-1.46)$ & $<0.01$ & $1.24(1.06-1.44)$ & $<0.01$ \\
\hline Tx & $1.22(1.03-1.45)$ & 0.02 & $1.21(1.02-1.45)$ & 0.03 \\
\hline \multicolumn{5}{|l|}{ AJCC.N } \\
\hline NO & Ref & & Ref & \\
\hline N1 & $0.879(0.74-1.04)$ & 0.13 & $0.86(0.72-1.04)$ & 0.12 \\
\hline N2 & $1.118(1.01-1.24)$ & 0.03 & $1.14(1.02-1.27)$ & 0.02 \\
\hline N3 & $1.13(0.99-1.28)$ & 0.05 & $1.22(1.07-1.40)$ & $<0.01$ \\
\hline Nx & $1.06(0.89-1.26)$ & 0.5 & $0.93(0.76-1.15)$ & 0.51 \\
\hline \multicolumn{5}{|c|}{ Chemoradiotherapy } \\
\hline None & Ref & & Ref & \\
\hline
\end{tabular}

Abbreviations: AC, adenocarcinoma; SSC, squamous carcinoma; NSCLC, non-small cell lung carcinoma, nothing otherwise special; Ref, reference. 


\begin{tabular}{|c|c|c|c|c|}
\hline \multirow[b]{2}{*}{ Radiation } & \multicolumn{2}{|l|}{ Univariate Analysis } & \multicolumn{2}{|c|}{ Multivariate Analysis } \\
\hline & $0.904(0.79-1.04)$ & 0.15 & $0.91(0.79-1.04)$ & 0.16 \\
\hline Chemotherapy & $0.482(0.40-0.57)$ & $<0.01$ & $0.48(0.40-0.57)$ & $<0.01$ \\
\hline Both & $0.518(0.46-0.59)$ & $<0.01$ & $0.51(0.44-0.58)$ & $<0.01$ \\
\hline \multicolumn{5}{|l|}{ Histology } \\
\hline AC & Ref & & Ref & \\
\hline SSC & $1.38(1.24-1.54)$ & $<0.01$ & $1.29(1.14-1.45)$ & $<0.01$ \\
\hline NSCLC & $1.32(1.18-1.48)$ & $<0.01$ & $1.25(1.10-1.41)$ & $<0.01$ \\
\hline \multicolumn{5}{|l|}{ Involvement } \\
\hline None & Ref & & Ref & \\
\hline Bone & $1.09(1.04-1.22)$ & 0.16 & $1.18(1.05-1.33)$ & $<0.01$ \\
\hline Liver & $1.43(1.17-1.76)$ & $<0.01$ & $1.42(1.15-1.76)$ & $<0.01$ \\
\hline Lung & $1.11(0.98-1.24)$ & 0.08 & $1.07(0.93-1.21)$ & 0.35 \\
\hline Bone + Liver & $1.30(1.09-1.55)$ & $<0.01$ & $1.35(1.10-1.65)$ & $<0.01$ \\
\hline Bone + Lung & $1.07(0.94-1.26)$ & 0.45 & $1.06(0.89-1.27)$ & 0.05 \\
\hline Liver + Lung & $1.46(1.16-1.85)$ & $<0.01$ & $1.47(1.17-1.85)$ & $<0.01$ \\
\hline All & $1.33(1.08-1.64)$ & $<0.01$ & $1.40(1.13-1.74)$ & $<0.01$ \\
\hline
\end{tabular}

\subsection{Subcategories of M1 cohort}

Based on independent prognostic factors from multivariable analysis of competing risk model, the eight groups of extracranial involvements were recombined into MA, MB and MC subcategories (Supplemental Table 2). The group with all three lesions topped the list, while the group without ECM ranked at the bottom. Given that the SHR of the other three groups with liver metastasis was higher than that of the bone solo group, they ranked higher. The two groups without independent prognosis ranked after the bone solo group. Only two of the seven $P$ values were $<0.05$, making the cutoff among MA, MB, and MC. The significance of subcategory prognostic difference was confirmed by SHR (MC VS. MB $1.15,95 \% \mathrm{Cl}$ 1.05-1.26; MB VS. MA 1.11, 95\%Cl 1.02-1.2).

\subsection{Multivariable analysis of the competing risk model}

Validation of the M1 subcategory system is described and depicted in supplemental Table 3. Multivariate analysis indicated that the MC and MB subcategories were risk factors in the training set (SHR, 1.097, 
$95 \% \mathrm{Cl}, 0.998-1.206, \mathrm{P}=0.05 ; \mathrm{SHR}, 1.392,95 \% \mathrm{Cl}, 1.232-1.573, \mathrm{P}<0.001)$, hold-out test set (SHR, 1.145, $95 \% \mathrm{Cl}, 1.04-1.261, \mathrm{P}=0.006$; SHR, 1.534, 95\% Cl, 1.363-1.725, $\mathrm{P}<0.001)$, and whole dataset (SHR, $1.126,95 \% \mathrm{Cl}, 1.053-1.205, \mathrm{P}<0.001$; SHR, $1.46,95 \% \mathrm{Cl}, 1.339-1.592, \mathrm{P}<0.001)$.

\subsection{Cumulative incidence curves of DSS and OS}

Cumulative incidence curves plotted the mortality augments across M1 subcategories in training, test and whole data sets. Again, the validation of the M1 subdivision was confirmed (Fig. 1).

\subsection{Subgroup analysis and interaction tests}

To study the interaction between treatment mortality and M1 subcategories, MB and MC were combined and compared to MA (Fig. 2). In the subgroups of no-chemoradiotherapy, radiotherapy, and chemoradiotherapy, presence of ECM was associated with unfavorable prognosis $(\mathrm{SHR}, 1.44,95 \% \mathrm{Cl}$, 1.21-1.71, $\mathrm{P}<0.01$; SHR, 1.22, 95\% Cl, 1.08-1.37, $\mathrm{P}<0.01$; SHR, 1.23, 95\% Cl, 1.07-1.41, $\mathrm{P}<0.01$, respectively).

Further analysis of interaction was applied, and significant interaction from the therapy regimen was observed. Compared to no-chemoradiotherapy, both chemotherapy and chemoradiotherapy have synergistic modification effects on ECM (adjusted SHR, 0.67, 95\% Cl, 0.67-0.81, P<0.01; adjusted SHR, $0.73,95 \% \mathrm{Cl}, 0.67-0.81, \mathrm{P}<0.01$, respectively), while solo radiotherapy imposed antagonistic modification effects on ECM (adjusted SHR, 1.25, 95\% Cl, 1.12-1.39, P<0.01).

\subsection{Kaplan-Meier curves and the Cox proportional hazard model}

Kaplan-Meier curves were depicted to investigate survival differences between the covariates (Fig. 3). Compared to MA, MB and MC were associated with increased risk for OS (Supplemental Table 4). HR of MB was $1.248(95 \% \mathrm{Cl}, 1.133-1.374), 1.203$ (95\% Cl, $1.094-1.323)$, and $1.226(95 \% \mathrm{Cl}, 1.146-1.312)$ in the training, test and whole datasets $(\mathrm{P}<0.001)$, while $\mathrm{HR}$ of $\mathrm{MC}$ was $1.892(95 \% \mathrm{Cl}, 1.685-2.124), 1.815$ $(95 \% \mathrm{Cl}, 1.612-2.044)$ and $1.856(95 \% \mathrm{Cl}, 1.709-2.016)$ in the training, test and whole datasets $(\mathrm{P}<$ $0.001)$.

\subsection{Construction and validation of a competing risk nomogram in the training set}

A competing risk nomogram was built via the Fine and Gray model to predict the 6-month, 12-month and 18-month cumulative death probabilities (Fig. 4A). Calculation details are listed in Supplemental Table 5.

As depicted in Fig. 4B, the calibration curves for DSS were close to the standard curves. The ROC curve used to assess the nomogram of DSS is shown in Fig. 5. AUC of DSS was $0.757,0.748$ and 0.738 for 6month, 12-month and 18-month prediction(Fig. 4C), respectively. DCA analysis proved the clinical value of this model for 6- and 12-month prediction. (Fig. 4D-F). 


\subsection{Validation of the nomogram in the hold-out test set}

The calibration curves for DSS in the hold-out test set were plot in Fig. 5A. Again, calibration curves were close to standard curves. The ROC curve used to assess the nomogram of DSS is shown in Fig. 5. AUC of DSS was $0.778,0.769$ and 0.756 for the 6 -month, 12-month, and 18-month prediction(Fig. 5B), respectively. The DCA analysis indicated the good value of this model for 6 - and 12-month predictions. (Fig. 5C-E).

\section{Discussion}

In the present study, the Fine and Gray proportional sub-distribution hazard model revealed that the anatomic extent of ECM is an independent prognostic factor for DSS in NSCLC with de novo BM. After recombination of ECM into three subtypes (MA, MB and MC), multivariable analysis based on the competing risk model was re-performed, with ECM subdivision, AJCC T and N, chemoradiotherapy, and histology found significantly associated with DSS. Furthermore, these independent predictive factors were employed to create 6-month, 12-month, and 18-month nomograms for DSS. While in the training cohort, the AUC for the 6-month DSS prediction reached 0.757 , it reached 0.778 in the internal validation cohort. The calibration curves did not deviate from the reference line. Decision curve analyses (DCA) revealed the net benefit of the nomogram for clinical utility.

The TNM ${ }^{8 \text { th }}$ classification of LC addressed the existence of synchronous oligo-metastatic patients with a single extrathoracic metastatic lesion (M1b), which is almost the same in prognosis to those presenting with the intra-thoracic metastatic disease only (M1a)[17]. In terms of prognosis, these two groups stood out from the other metastatic population (M1c), which involved multiple metastatic organs. Identification of this new category can be related to several factors, including improvement in diagnostic and imaging accuracy, the continuous increase in systemic oncological treatment efficacy, and the favorable tolerability of local ablative treatments[18]. Given the outcome variability within M1c stages, identifying independent, but complementary prognostic factors is of intense interest[19]. As mentioned in the introduction, various models have been proposed to prognosticate patients with brain metastasis[20-22]. The DS-GPA incorporates the presence/ absence of ECM, but a wide range of individualized survival probability was found within each DS-GPA class. In addition to its presence alone, more ECM lesions were associated with a poorer prognosis in NSCLC patients with BM[9, 23].

According to our analyses, ECM patterns in NSCLC with BM is qualified to stratify prognosis. Patients were divided into MA (neither liver, bone, nor lung involvement), MB (without liver involvement), and MC (with liver involvement). The MA subcategory had relatively favorable prognosis, while the MC subcategory had significant survival disadvantage. Our stratification is consistent with previous research $[9,24]$. It has been proposed that distant metastases in LC are nonrandom and there may be specific patterns of distant metastases[25]. According to the seed and soil hypothesis[26], there are complex ways for disseminated cells to interact with the host organ microenvironment, hence different interactions may result in distinctive patterns of metastatic events. According to our analyses, once the 
liver becomes involved, patient survival would be impaired. One reported theory is that, as the liver is an immunosuppressive organ when metastases occur, liver metastasis impedes the liver's immune surveillance of other ongoing metastases[27]. Similarly, NSCLC patients with metastasis to their brain and liver were more likely to progress when treated with PD-1 inhibitors.

Multivariable analysis of the competing risk model, as well as $\mathrm{CIF}$, validated the independent prognostic role of ECM subdivision in NSCLC with de novo BM. In a cohort of 4974 patients, our analysis harbored a large number of NSCLC cases with initial BM, hence enough statistical power to examine the potential correlation between ECM subdivision and DSS. The competing risk model was somehow different from the Cox model, suggesting that the improvement in the competing risk model was relevant. Elderly LC patients with BM have disadvantages in various aspects, such as immuno-senescence and decreased physiologic reserve[28]. The competing risk model can improve stratification of elderly cancer patients with cancer-specific death to overall mortality $[15,29]$. Although the competing risk model was less than perfect, it was validated in our data.

The nomogram allowed for individualized estimation of DSS and highlighted the potential to tailor predictions to individual patients rather than group-wise estimations from other models like the DS-GPA. This nomogram can be readily used in clinical practice to provide patients and their physician with an individualized survival estimate, using readily available variables. As early as 2012 , Jill S proposed a nomogram for individual estimation of survival among patients with BM. They incorporated age, KPS, primary sites, histology, status of primary disease, metastatic spread (brain alone or with ECM), and number of brain lesions. After direct comparison with the DS-GPA, our nomogram was superior in individualized estimations of survival. Compared to Jill S' nomogram, our nomogram can provide an even more detailed prognosis for elderly NSCLC patients with BM, although direct comparison between these two nomograms wasn't available due to lack of relevant information in SEER. Another nomogram was plotted by Heng Shen to predict early death of LC patients with synchronous BM[30]. Age, race, gender, Gleason grade, histological type, T stage, $\mathrm{N}$ stage, bone metastasis, liver metastasis and marital status were included in the nomogram. The AUC of the ROC curve was 0.794 (95\% Cl: 0.788-0.799). Similarly, in Heng Shen's nomogram, synchronous liver metastasis in LC patients with BM significantly increases the risk of early death. Hence, this nomogram is consistent with ours.

This study had several limitations. The demographic and clinical information provided by SEER isn't complete.It also lacks detailed records of treatment, such as chemotherapy and radiotherapy. Both targeted therapy and immunotherapy have become popular in the comprehensive treatment of NSCLC with BM. However, the SEER database has neither specific information about these novel therapies nor mutation data. Third, head-to-head comparisons between our nomogram and the DS-PGA were unavailable because DS-PGA couldn't be calculated based on SEER data. Additionally, we used internal samples to validate the competing risk nomogram. It would be better if external validation from another dataset was available. Thus, more studies are necessary to validate our results in the future.

\section{Conclusion}


In conclusion, the extracranial subdivision of $\mathrm{M} 1$ categories harbors the type and number of metastatic sites and provides extra information for prognosis in septuagenarians and octogenarians with De Novo Brain-metastatic NSCLC. A created competing risk nomogram incorporated the pattern of ECM, AJCC T and $\mathrm{N}$, histology, and chemoradiotherapy. This may act as a useful implement for clinicians to evaluate DSS and help to choose appropriate medical care.

\section{Declarations}

\section{Author contributions}

Mingwei Zhang and Xiaoping Chen conceived and designed the study. Zijing Zhu analyzed the data. Wenying Jiang authored the manuscript. Shaoli Peng and Weitong Zhou prepared the figures and tables. Jinfu Zhuang supervised the study. Jinsheng Hong revised the manuscript. All authors reviewed the final manuscript.

\section{Acknowledgement}

This study was supported by National Natural Science Foundation of China (82003386 and U1805263); the Special Projects of the Central Government Guiding Local Science and Technology Development (2021 L3018); Natural Science Foundation of Fujian Province (2021J01658); the program for Probability and Statistics: Theory and Application (IRTL1704) and Innovative Research Team in Science and Technology in Fujian Province University (IRTSTFJ); Fujian Provincial Finance Project (No.2019B032).

\section{Declaration of competing interest: None.}

\section{References}

1. Sung H, Ferlay J, Siegel RL, Laversanne M, Soerjomataram I, Jemal A, Bray F (2021) Global Cancer Statistics 2020: GLOBOCAN Estimates of Incidence and Mortality Worldwide for 36 Cancers in 185 Countries. Cancer J Clin 71(3):209-249. https://doi.org/10.3322/caac.21660

2. Karimpour M, Ravanbakhsh R, Maydanchi M, Rajabi A, Azizi F, Saber A (2021) Cancer driver gene and non-coding RNA alterations as biomarkers of brain metastasis in lung cancer: A review of the literature. Biomed Pharmacother 143:112190. https://doi.org/10.1016/j.biopha.2021.112190

3. Lei L, Wang W-X, Wang D, Lin L, Zhu Y-C, Wang H, Wang L-P, Zhuang W, Fang M-Y, Wan B, Feng H-J, Xu C-W (2021) A real-world study in advanced non-small cell lung cancer with de novo brain metastasis. J Cancer 12(5):1467-1473. .https://doi.org/10.7150/jca.51411

4. Cacho-Díaz B, Cuapaténcatl LD, Rodríguez JA, Garcilazo-Reyes YJ, Reynoso-Noverón N, Arrieta 0 (2021) Identification of a high-risk group for brain metastases in non-small cell lung cancer patients. J Neurooncol. https://doi.org/10.1007/s11060-021-03849-w

5. Schoenmaekers JJAO, Dursun S, Biesmans C, De Ruysscher DKM, Broen MPG, Remon J, Dingemans A-MC, Hendriks LEL (2021) Dynamics of eligibility criteria for central nervous system metastases in 
non-small cell lung cancer randomized clinical trials over time: A systematic review. Crit Rev Oncol Hematol 166:103460. https://doi.org/10.1016/j.critrevonc.2021.103460

6. Li J, Jing W, Zhai X, Jia W, Zhu H, Yu J (2021) Estimating Survival in Patients with Non-Small-Cell Lung Cancer and Brain Metastases: A Verification of the Graded Prognostic Assessment for Lung Cancer Using Molecular Markers (Lung-molGPA). OncoTargets and therapy 14:1623-1631. .https://doi.org/10.2147/OTT.S288928

7. Schag CC, Heinrich RL, Ganz PA (1984) Karnofsky performance status revisited: reliability, validity, and guidelines. J Clin oncology: official J Am Soc Clin Oncol 2(3):187-193. https://pubmed.ncbi.nlm.nih.gov/6699671

8. Lin C, Wu J, Ding S, Goh C, Andriani L, Lu S, Shen K, Zhu L (2019) Subdivision of M1 Stage for De Novo Metastatic Breast Cancer to Better Predict Prognosis and Response to Primary Tumor Surgery. J Natl Compr Cancer Network: JNCCN 17(12):1521-1528. .https://doi.org/10.6004/jnccn.2019.7332

9. Wang M, Wu Q, Zhang J, Qin G, Yang T, Liu Y, Wang X, Zhang B, Wei Y (2021) Prognostic impacts of extracranial metastasis on non-small cell lung cancer with brain metastasis: A retrospective study based on surveillance, epidemiology, and end results database. Cancer Med 10(2):471-482. https://doi.org/10.1002/cam4.3562

10. Meoni G, Cecere FL, Lucherini E, Di Costanzo F (2013) Medical treatment of advanced non-small cell lung cancer in elderly patients: a review of the role of chemotherapy and targeted agents. $\mathrm{J}$ geriatric Oncol 4(3):282-290. https://doi.org/10.1016/j.jgo.2013.04.005

11. Thomas P-A, Couderc A-L, Boulate D, Greillier L, Charvet A, Brioude G, Trousse D, D'Journo X-B, Barlesi F, Loundou A (2021) Early-stage non-small cell lung cancer beyond life expectancy: Still not too old for surgery? Lung Cancer 152:86-93. https://doi.org/10.1016/j.lungcan.2020.12.009

12. Lamba N, Kearney RB, Catalano PJ, Hassett MJ, Wen PY, Haas-Kogan DA, Aizer AA (2021) Population-based estimates of survival among elderly patients with brain metastases. Neuro Oncol 23(4):661-676. https://doi.org/10.1093/neuonc/noaa233

13. Dohm A, Diaz R, Nanda RH (2021) The Role of Radiation Therapy in the Older Patient. Curr Oncol Rep 23(1):11. https://doi.org/10.1007/s11912-020-01000-y

14. WHO. Global Health Estimates (2020) : Deaths by Cause, Age, Sex, by Country and by region, 20002019. whoint/ data/gho/data/theme s/morta lity-andgloba I-healt h-estim ates/ghe-leadi ngcause sof-death. 2020, who.int/ data/gho/data/theme s/morta lity-andgloba I-healt h-estim ates/ghe-leadi ngcause s-of-death

15. Carmona R, Zakeri K, Green G, Hwang L, Gulaya S, Xu B, Verma R, Williamson CW, Triplett DP, Rose BS, Shen H, Vaida F, Murphy JD, Mell LK (2016) Improved Method to Stratify Elderly Patients With Cancer at Risk for Competing Events. J Clin oncology: official J Am Soc Clin Oncol 34(11):12701277. .https://doi.org/10.1200/JC0.2015.65.0739

16. Lamba N, Wen PY, Aizer AA (2021) Epidemiology of brain metastases and leptomeningeal disease. Neuro Oncol 23(9):1447-1456. https://doi.org/10.1093/neuonc/noab101 
17. Eberhardt WEE, Mitchell A, Crowley J, Kondo H, Kim YT, Turrisi A, Goldstraw P, Rami-Porta R (2015) The IASLC Lung Cancer Staging Project: Proposals for the Revision of the M Descriptors in the Forthcoming Eighth Edition of the TNM Classification of Lung Cancer. J Thorac Oncol 10(11):15151522. .https://doi.org/10.1097/JT0.0000000000000673

18. deSouza NM, Liu Y, Chiti A, Oprea-Lager D, Gebhart G, Van Beers BE, Herrmann K, Lecouvet FE (2018) Strategies and technical challenges for imaging oligometastatic disease: Recommendations from the European Organisation for Research and Treatment of Cancer imaging group. Eur $\mathrm{J}$ Cancer 91:153-163. https://doi.org/10.1016/j.ejca.2017.12.012

19. Smeltzer MP, Faris NR, Ray MA, Osarogiagbon RU (2018) Association of Pathologic Nodal Staging Quality With Survival Among Patients With Non-Small Cell Lung Cancer After Resection With Curative Intent. JAMA Oncol 4(1):80-87. https://doi.org/10.1001/jamaoncol.2017.2993

20. Gaspar L, Scott C, Rotman M, Asbell S, Phillips T, Wasserman T, McKenna WG, Byhardt R (1997) Recursive partitioning analysis (RPA) of prognostic factors in three Radiation Therapy Oncology Group (RTOG) brain metastases trials. Int J Radiat Oncol Biol Phys 37(4):745-751. https://pubmed.ncbi.nlm.nih.gov/9128946

21. Sperduto PW, Kased N, Roberge D, Xu Z, Shanley R, Luo X, Sneed PK, Chao ST, Weil RJ, Suh J, Bhatt A, Jensen AW, Brown PD, Shih HA, Kirkpatrick J, Gaspar LE, Fiveash JB, Chiang V, Knisely JPS, Sperduto CM, Lin N, Mehta M (2012) Summary report on the graded prognostic assessment: an accurate and facile diagnosis-specific tool to estimate survival for patients with brain metastases. $J$ Clin oncology: official J Am Soc Clin Oncol 30(4):419-425. https://doi.org/10.1200/JC0.2011.38.0527

22. Sperduto PW, Yang TJ, Beal K, Pan H, Brown PD, Bangdiwala A, Shanley R, Yeh N, Gaspar LE, Braunstein S, Sneed P, Boyle J, Kirkpatrick JP, Mak KS, Shih HA, Engelman A, Roberge D, Arvold ND, Alexander B, Awad MM, Contessa J, Chiang V, Hardie J, Ma D, Lou E, Sperduto W, Mehta MP (2017) Estimating Survival in Patients With Lung Cancer and Brain Metastases: An Update of the Graded Prognostic Assessment for Lung Cancer Using Molecular Markers (Lung-molGPA). JAMA Oncol 3(6):827-831. https://doi.org/10.1001/jamaoncol.2016.3834

23. Higuera Gómez O, Moreno Paul A, Ortega Granados AL, Ros Martínez S, Pérez Parente D, Ruiz Gracia P, Sáenz Cuervo-Arango L, Vilà L (2021) "High Tumor Burden" in Metastatic Non-Small Cell Lung Cancer: Defining the Concept. Cancer Manage Res 13:4665-4670.

.https://doi.org/10.2147/CMAR.S302928

24. Li J, Zhu H, Sun L, Xu W, Wang X (2019) Prognostic value of site-specific metastases in lung cancer: A population based study. J Cancer 10(14):3079-3086. .https://doi.org/10.7150/jca.30463

25. Huang Y, Zhu L, Guo T, Chen W, Zhang Z, Li W, Pan X (2021) Metastatic sites as predictors in advanced NSCLC treated with PD-1 inhibitors: a systematic review and meta-analysis. Hum Vaccin Immunother 17(5):1278-1287. . https://doi.org/10.1080/21645515.2020.1823779

26. Belluomini L, Dodi A, Caldart A, Kadrija D, Sposito M, Casali M, Sartori G, Ferrara MG, Avancini A, Bria E, Menis J, Milella M, Pilotto S (2021) A narrative review on tumor microenvironment in 
oligometastatic and oligoprogressive non-small cell lung cancer: a lot remains to be done. Transl Lung Cancer Res 10(7):3369-3384. .https://doi.org/10.21037/tlcr-20-1134

27. Fan Z, Huang Z, Tong Y, Zhu Z, Huang X, Sun H (2021) Sites of Synchronous Distant Metastases, Prognosis, and Nomogram for Small Cell Lung Cancer Patients with Bone Metastasis: A Large Cohort Retrospective Study. J Oncol. 2021:9949714.https://doi.org/10.1155/2021/9949714

28. Gu Y, Zhang J, Zhou Z, Liu D, Zhu H, Wen J, Xu X, Chen T, Fan M (2020) Metastasis Patterns and Prognosis of Octogenarians with NSCLC: A Population-based Study. Aging Dis 11(1):82-92. https://doi.org/10.14336/AD.2019.0414

29. Dignam JJ, Kocherginsky MN (2008) Choice and interpretation of statistical tests used when competing risks are present. J Clin oncology: official J Am Soc Clin Oncol 26(24):4027-4034. .https://doi.org/10.1200/JC0.2007.12.9866

30. Shen H, Deng G, Chen Q, Qian J (2021) The incidence, risk factors and predictive nomograms for early death of lung cancer with synchronous brain metastasis: a retrospective study in the SEER database. BMC Cancer 21(1):825. https://doi.org/10.1186/s12885-021-08490-4

\section{Figures}

a

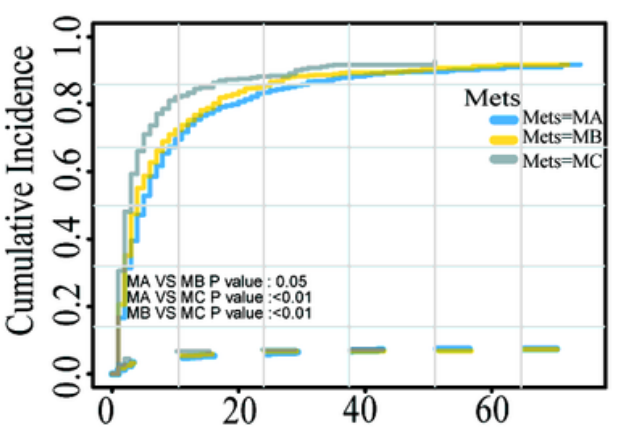

Time(months)

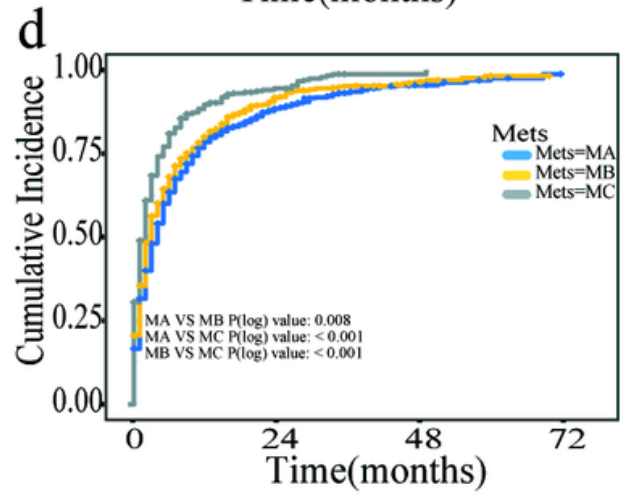

b

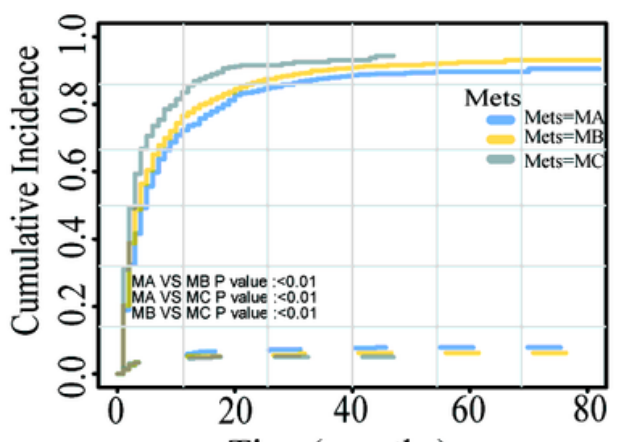

Time(months)

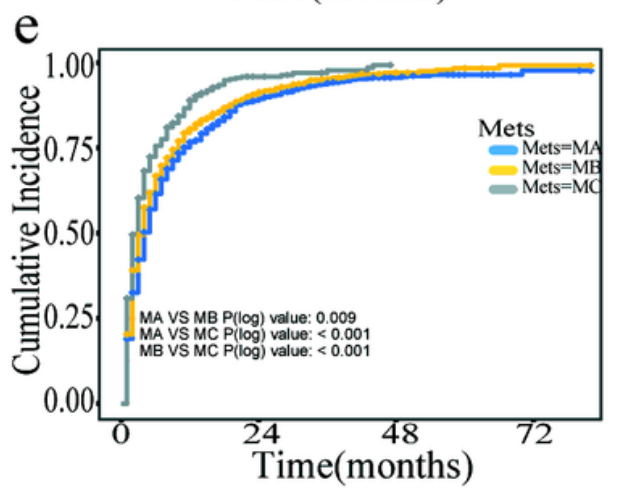

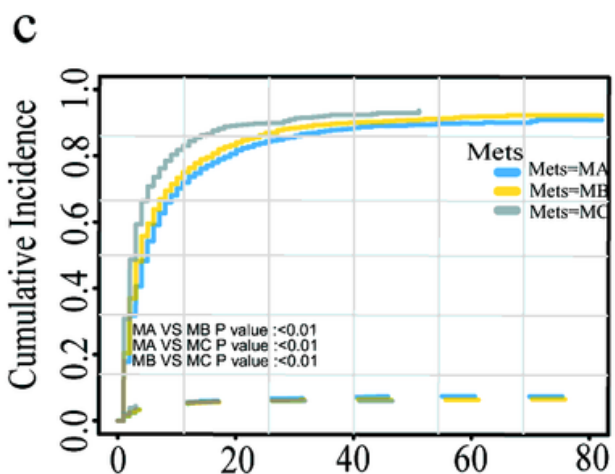

Time(months)

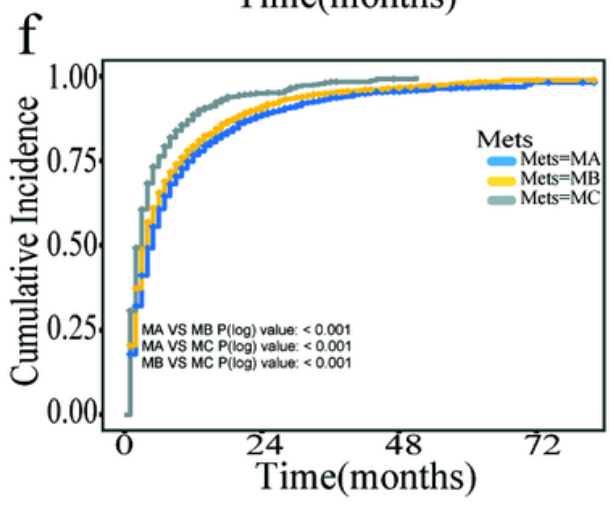

\section{Figure 1}

Curves of Cumulative Incidence (A-C) and Log-rank Test (D-F) among ECM patterns: the training set $(A \& D)$, the hold-out test set (B\&E), and the whole set (C\&F). 
a

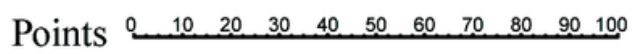

AJCC.T $\frac{\mathrm{T}^{2}}{\mathrm{~T}_{1} \mathrm{~T}_{\mathrm{T}}} \mathrm{T}_{\mathrm{T}}$

AJCC.N $\frac{\mathrm{Nx}}{\sqrt{0} \frac{\mathrm{N} 1}{\mathrm{~N}} \mathrm{~N}_{3}}$

Histology $\frac{\mathrm{NSCLC}}{\mathrm{AC} \quad \mathrm{SSC}}$

Chemoradiotherapy $\frac{3, \text { Both }}{2 \text { C None }}$

Mets $\frac{\mathrm{MB}}{\mathrm{MA}}$

Total Points 0 20 406080100120140160180200220240260

6-month DSS $0.8 \quad \begin{array}{llllll}6 & 0.6 & 0.4 & 0.2 & 0.1\end{array}$

12-month DSS $\begin{array}{lllll}0.6 & 0.4 & 0.2 & 0.1\end{array}$

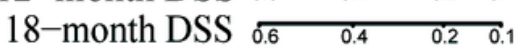

d

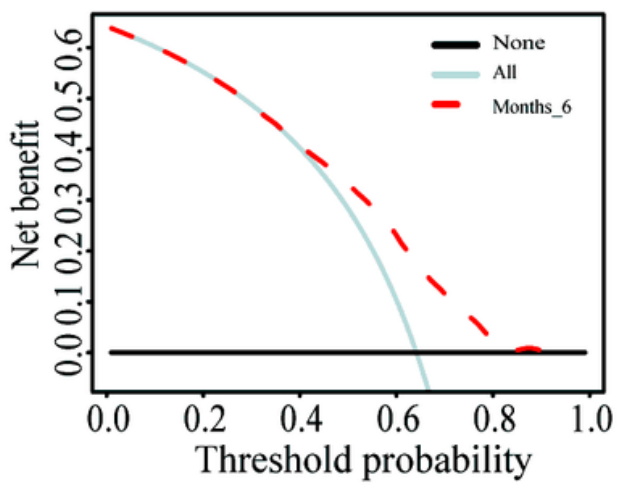

b

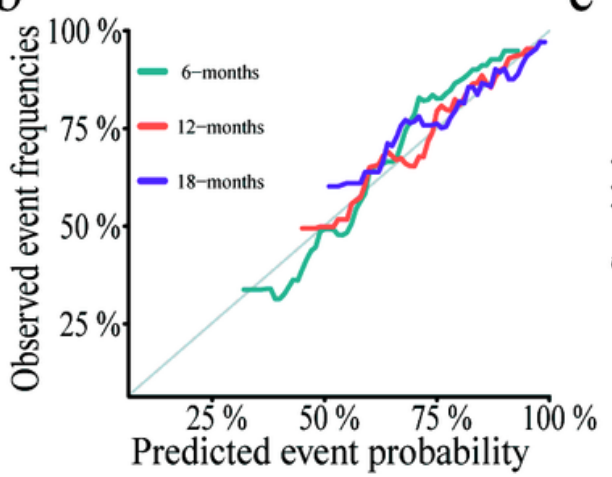

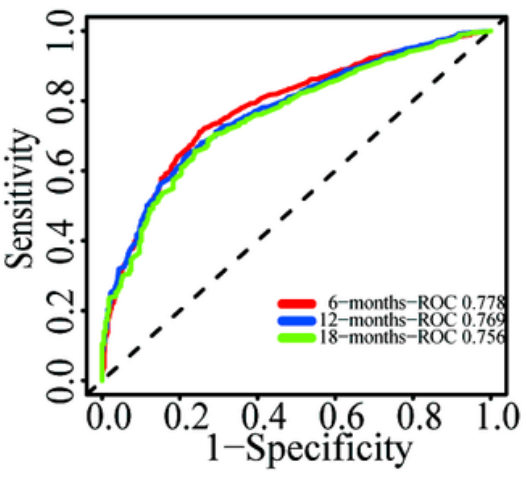

e

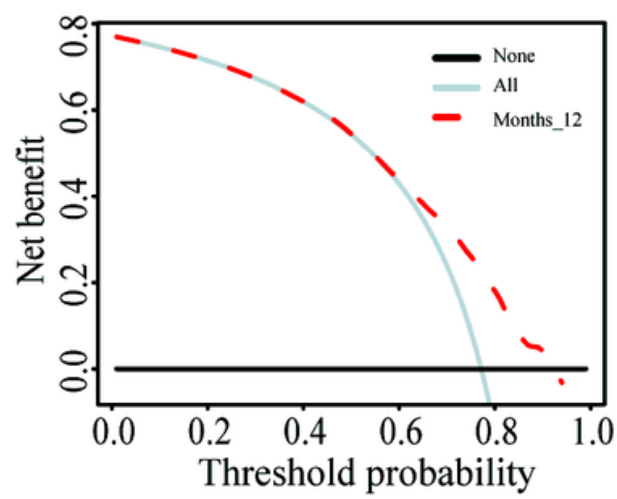

$\mathrm{f}$

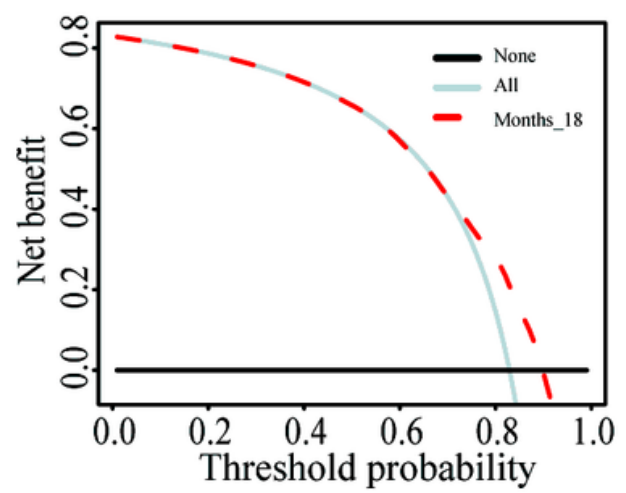

Figure 2

Competing risk nomogram predicting 6-month, 12-month, and 18-month cumulative probabilities of DSS in elderly NSCLC patients with synchronous BM (A), calibration curves and ROC curves with AUC for 6month, 12-month, and 18-month prediction (B and C), DCA curves for 6-month (D), 12-month (E), and 18month $(F)$ prediction, in the training set.

Abbreviations: AC, adenocarcinoma; SSC, squamous carcinoma; NSCLC , non-small cell lung carcinoma, nothing otherwise special; Mets subdivision, extracranial metastasis subdivision. 
a

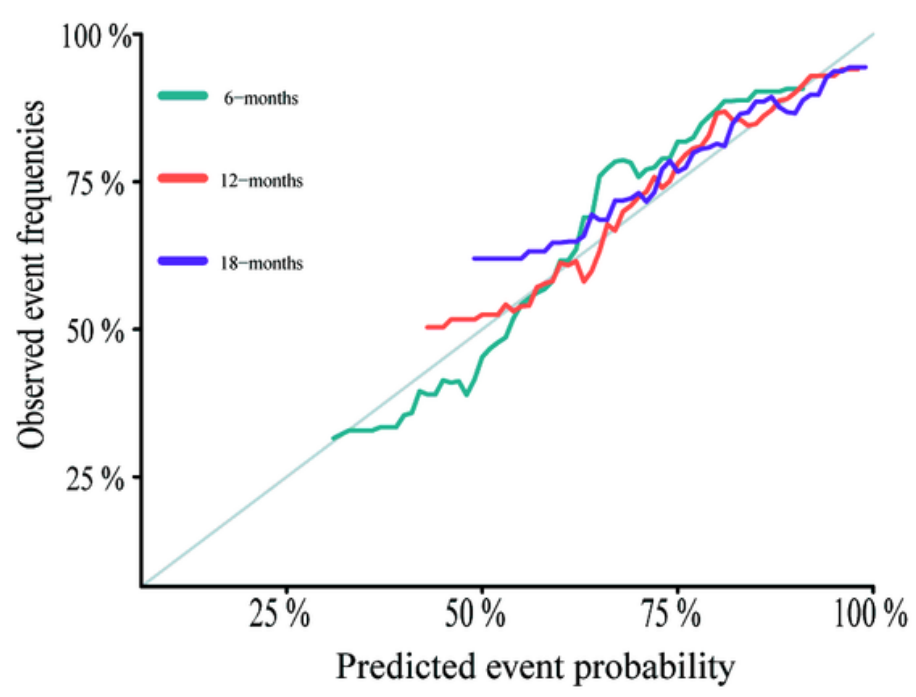

c

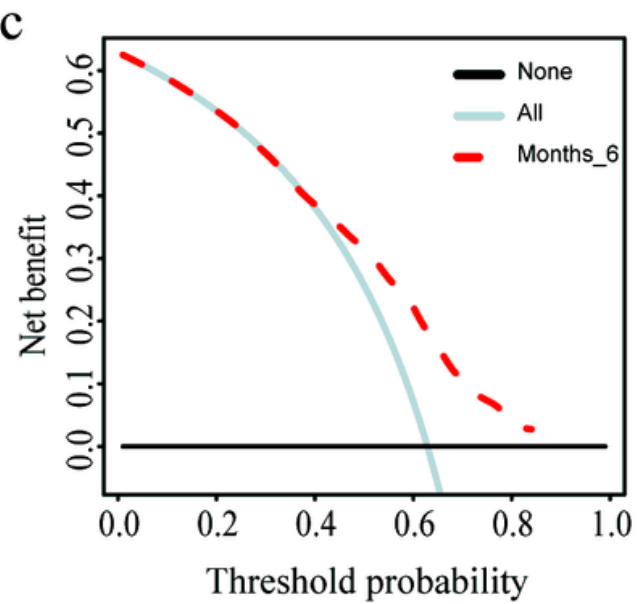

$\mathrm{b}$
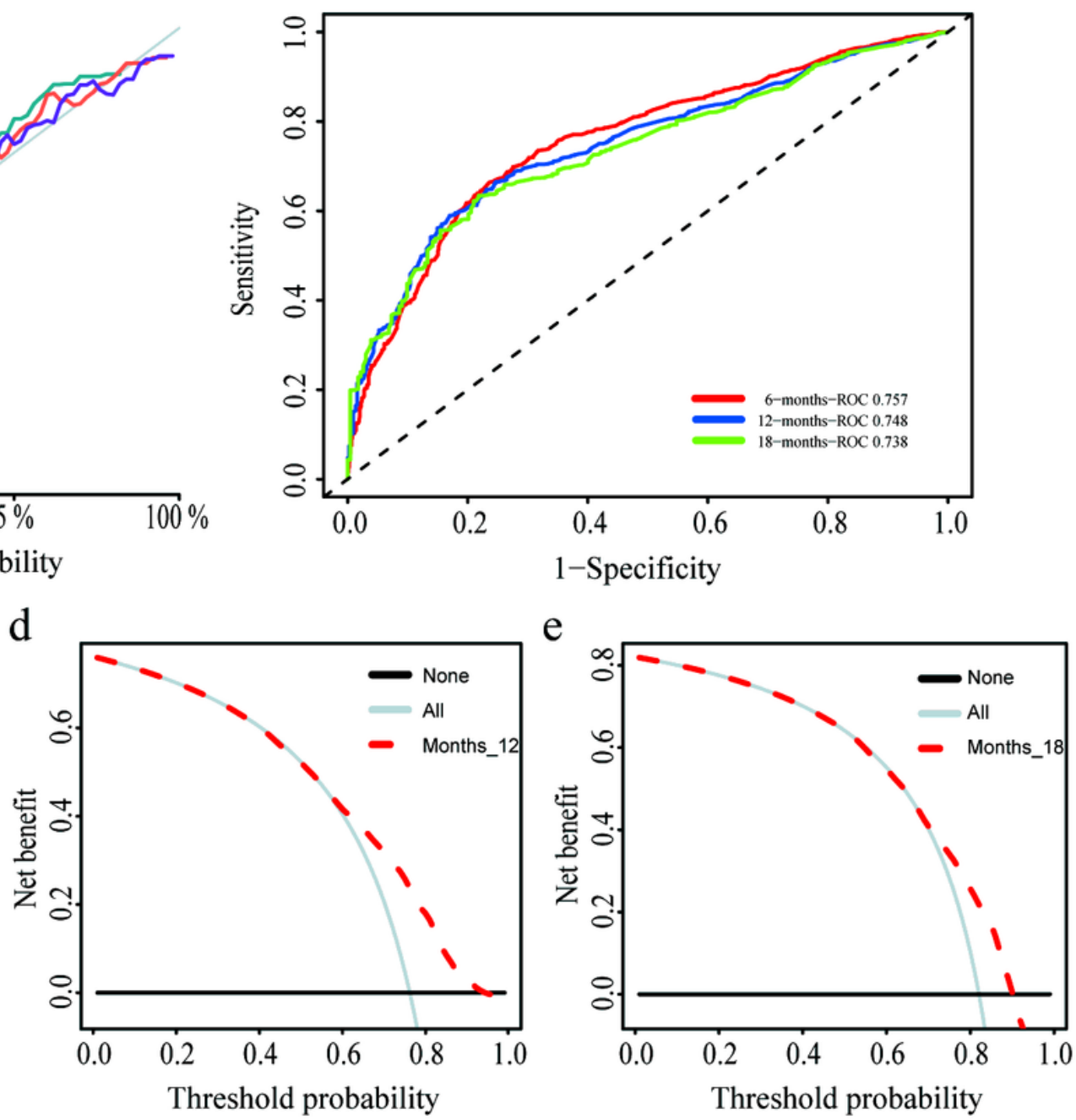

Figure 3

Validation of the nomogram in the hold-out test set: calibration curves and ROC curves with AUC for 6month, 12-month, and 18-month prediction (A and B), DCA curves for 6-month (C), 12-month (D), and 18month $(E)$ prediction, in the hold-out test set.

\section{Supplementary Files}

This is a list of supplementary files associated with this preprint. Click to download.

- S1table.docx

- S2table.docx

- S3table.docx

- S4table.docx

- S5table.docx

- Supplementalfigure1.tif 
- Supplementalfigure2.tif

Page 20/20 